Document downloaded from:

http://hdl.handle.net/10251/79450

This paper must be cited as:

Ferrando Martín, V.; Remón Martín, L.; Pons Martí, A.; Furlan, WD.; Monsoriu Serra, JA. (2016). Wavefront sensing using a graphical user interface. Computer Applications in Engineering Education. 24(2):255-262. doi:10.1002/cae.21703.

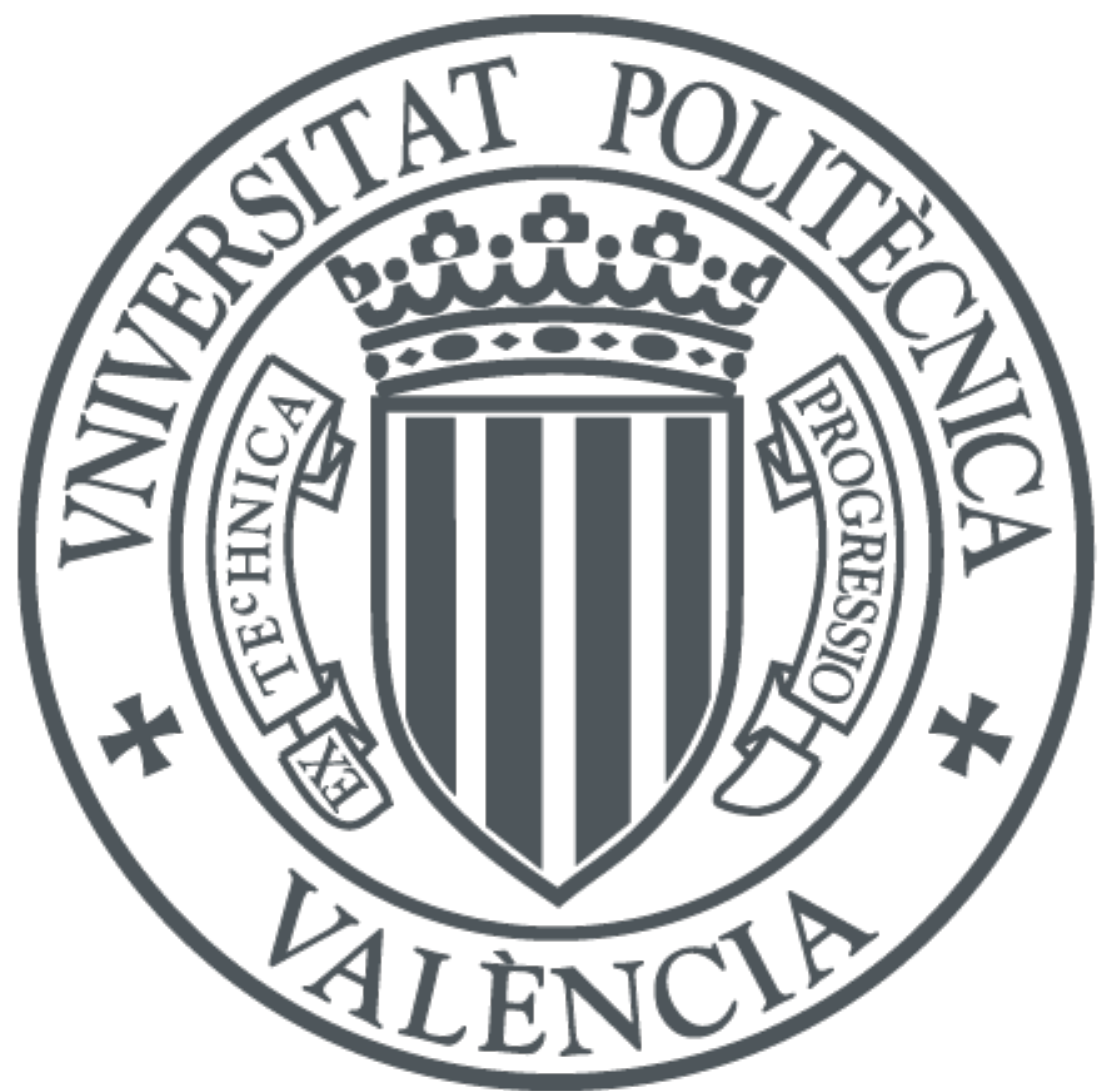

The final publication is available at

http://dx.doi.org/10.1002/cae.21703

Copyright Wiley

Additional Information 


\title{
Wavefront sensing using a Graphical User Interface
}

\author{
Vicente Ferrando ${ }^{1,2}$, Laura Remón ${ }^{1}$, Amparo Pons ${ }^{2}$, \\ Walter D. Furlan ${ }^{2}$ and Juan A. Monsoriu ${ }^{1, *}$ \\ ${ }^{1}$ Centro de Tecnologías Físicas, Universitat Politècnica de València, 46022 Valencia, Spain. \\ ${ }^{2}$ Departamento de Óptica, Universitat de València, 46100 Burjassot, Spain. \\ "E-mail: jmonsori@fis.upv.es
}

\begin{abstract}
We present an open wavefront sensor setup assisted with MATLAB programming to be used to teach the operating principle of Shack-Hartmann aberrometry. A new Graphical User Interface (GUI) has also been developed to determine the wavefront parameters from experimental measurements and the associated aberrations, which is a fundamental issue in Optical Engineering. From a didactical point of view, the proposed method allows students to interpret the results in a visual and heuristic way.
\end{abstract}

Key words: Wavefront sensor, Aberrometer, Optical teaching.

\section{Introduction}

Wavefront sensors, also known as aberrometers, are important instruments in Optics and Wave Physics in general. For example, these instruments are commonly used in Adaptative Optics to improve the images taken with modern astronomical telescopes by reducing the effect of wavefront distortions introduced by the earth's atmosphere [1]. Wavefront sensors have been also used extensively in various research fields, including optical communications [2], microscopy [3], fiber lasers [4], singular optics [5], and eye surgery [6], among others. 
Several types of wavefront sensors have been efficiently developed, including wavefront curvature sensor [7], pyramid wavefront sensor [8], common-path interferometer [9], wavelet-transform profilometry [10], or shearing interferometer [11]. However, ShackHartmann (SH) based devices are probably the most popular [12, 13]. Its principle is relatively simple and well known [14]. It consists on an array of microlenses of the same focal length located in front of a camera sensor. The local tilt of the wavefront across each lens can then be calculated from the position of the focal spot on the sensor. The complete wavefront is then derivated using a reconstruction algorithm based, for instance, on Zernike polynomial decomposition [15].

Since a SH wavefront sensor is a very important device in science and technology, it should be taught to students in Optics and Wave Physics courses. Incorporating a SH sensor into optics laboratories, students are introduced to contemporary research activities. Unfortunately, SH sensors from laboratory suppliers are extremely expensive for pedagogical purposes. Moreover, commercial SH sensors are closed units in which is not possible to investigate the influence of the different internal components. Other wavefront sensors have been developed for its use in undergraduate laboratories, but these setups incorporate unconventional components, as for example arrays of micro zone plates [16]. This kind of array of diffractive lenses can be designed in a computer and can be printed on a transparent film. However, a binary zone plate is only able to focus the $10 \%$ of the incident light. Another alternative is to replace the array of microlenses by a simple pinhole array, but with significant light losses.

In this work we present a low cost SH sensor developed with the standard equipment present in most undergraduate optics laboratories. The proposed open experimental setup is assisted with MATLAB programming. MATLAB has emerged as a common programming language for science and technology due to its ease of use and flexibility [17-22]. We have developed a new Graphical User Interface (GUI) that allows students to obtain a rapid reconstruction of the wavefront from experimental measurements by using the Zernike polynomials. In particular, we have used the customized SH sensor as a focimeter to 
characterize different ophthalmic lenses. The GUI also provides the lens high order aberrations which is essential to determine the quality of this kind of lenses.

\section{Basic theory}

The principle of operation of the SH wavefront sensor is shown in Fig. 1. The two basic elements of the sensor are a Microlens Array (MLA) and a CCD as a camera sensor. A plane wave incident upon the device produces an array of spots on a regular matrix determined by the geometry of the lenslet array. An aberrated wavefront gives an array of displaced spots, being the centroid of each spot determined by the average slope of the wavefront over each lenslet. The centroid of theses spots can be found by applying object detection algorithms. The wavefront slope at each point can be derived from the relation between the displacements of the spots with the partial wavefront derivatives [14]:

$$
\begin{aligned}
& \frac{\partial W(\bar{x}, \bar{y})}{\partial x}=\frac{\Delta x(\bar{x}, \bar{y})}{f_{M L A}}, \\
& \frac{\partial W(\bar{x}, \bar{y})}{\partial y}=\frac{\Delta y(\bar{x}, \bar{y})}{f_{M L A}},
\end{aligned}
$$

where $[\bar{x}, \bar{y}]$ are the reference spots position vector, $W(\bar{x}, \bar{y})$ is the Aberrated Wavefront (AW), $f_{M L A}$ is the focal distance of the MLA, and $[\Delta x(\bar{x}, \bar{y}), \Delta y(\bar{x}, \bar{y})]$ are the displacements vector in the $x$ direction and $y$ direction respectively.

The AW can be expanded in the orthonormal Zernike basis [23]. The pupils are usually circular and this basis is orthonormal over the unit circle. Then, the wavefront phase can be expressed as [24]

$$
W(\bar{x}, \bar{y})=\sum_{i=1}^{M} a_{i} Z_{i}(\bar{x}, \bar{y}),
$$

where $M$ is the number of Zernike modes employed in the decomposition, $Z_{i}(\bar{x}, \bar{y})$ is the Zernike function for the $i^{\text {th }}$ mode, and $a_{i}$ is the $i^{\text {th }}$ Zernike coefficient. Each Zernike polynomial represents a particular wavefront aberration and the coefficients represent the weight of each Zernike term. Applying Eq. (2) to Eq. (1) we obtain: 


$$
\begin{aligned}
& \frac{\Delta x(\bar{x}, \bar{y})}{f_{M L A}}=\sum_{i=1}^{M} a_{i} \frac{\partial Z_{i}(\bar{x}, \bar{y})}{\partial x}, \\
& \frac{\Delta y(\bar{x}, \bar{y})}{f_{M L A}}=\sum_{i=1}^{M} a_{i} \frac{\partial Z_{i}(\bar{x}, \bar{y})}{\partial y} .
\end{aligned}
$$

The above expression can be rewritten in a matrix form as

$$
\frac{1}{f_{M L A}} \cdot \boldsymbol{d}=\boldsymbol{B} \cdot \boldsymbol{a},
$$

where $\boldsymbol{d}$ is a vector of dimension $2 N \times 1$ containing the displacements, $N$ is the number of microlenses that sample the wavefront, and $\boldsymbol{a}$ is the Zernike coefficients vector of dimension $M \times 1$ with the Zernike coefficients. The matrix $\boldsymbol{B}$, with the derivates of the Zernike functions, has dimension $2 N \times M$.

In order to obtain the Zernike coefficients vector, $\boldsymbol{a}$, we have to invert $\boldsymbol{B}$. Taking into account that $\boldsymbol{B}$ is, in general, a rectangular matrix, the left-sided inverse matrix is used for this purpose, obtaining

$$
\boldsymbol{a}=\left(\boldsymbol{B}^{\mathrm{T}} \cdot \boldsymbol{B}\right)^{-1} \cdot \boldsymbol{B}^{\mathrm{T}} \cdot \boldsymbol{d}
$$

For the particular case of an ophthalmic sphero-cylindrical lens without high order aberrations the surfaces are quadratic sections. Then, the generated wavefront under planewave illumination can be expressed as:

$$
W(\bar{x}, \bar{y})=a_{3} Z_{3}(\bar{x}, \bar{y})+a_{4} Z_{4}(\bar{x}, \bar{y})+a_{5} Z_{5}(\bar{x}, \bar{y}) .
$$

From these Zernike polynomials of the second order, the refractive error can be calculated using a least squares fitting of the wavefront [25]. The Zernike coefficients can be converted to a sphero-cylindrical ophthalmic notation through the equations [26]:

$$
C=-\frac{4}{R_{P}^{2}} \sqrt{6\left(a_{3}^{2}+a_{5}^{2}\right)} \quad S=-\frac{4 \sqrt{3} a_{4}}{R_{\mathrm{P}}^{2}}-\frac{C}{2} \quad \alpha=\frac{1}{2} \operatorname{atan}\left(\frac{a_{3}}{a_{5}}\right),
$$

where $R_{\mathrm{P}}$ is the pupil radius, $S$ is the spherical power of the lens in dioptres (D), $C$ is the cylindrical power (astigmatism) of the lens in diopters (D), and $\alpha$ corresponds to the axis of the cylindrical power in degrees $\left({ }^{\circ}\right)$. 


\section{The program}

We have developed a new MATLAB GUI for a rapid reconstruction of the wavefront produced by different tested lenses. Fig. 2 shows the algorithm flowchart of the program:

- First, the user has to introduce the input parameters (as detailed in Sect.3.1).

- The program displays both images and obtains the reference and test focal spots positions, which allows calculating the displacements vector $(\mathbf{d})$.

- Using the matrix with the derivatives of the Zernike functions (B) computed at the reference spots positions and the above displacements vector (d), the Zernike coefficients can be obtained with the Eq. (5).

- Finally, the program performs a wavefront reconstruction of the incident beam using Eq. (2) with the calculated Zernike coefficients and determines the spherocylindrical ophthalmic parameters using Eq. (7).

Following this flowchart, we have developed a Graphical User Interface (GUI) which main screen is shown in Fig. 3. The interface is structured in three parts: The input parameters screen on the left, the calculation screen at the center, and the output screen on the right.

\subsection{The input parameters screen}

Fig. 4 shows the screen corresponding to the input parameters that need to be provided:

- The reference and test image images are loaded by selecting the corresponding files (Reference.jpg and Lens4_1.jpg, respectively, in the example of Fig. 4).

- The parameter $f_{M L A}$ must be adjusted to the characteristics of the MLA implemented ( $f_{M L A}=50 \mathrm{~mm}$ in our laboratory).

- Other parameters such as the pixel size in microns of the loaded images ( $\Delta_{p i x}=4.4 \mu \mathrm{m}$ in the example of Fig. 4) and the wavelength of the lighting source ( $\lambda=633 \mathrm{~nm}$ in the example of Fig. 4) have to be introduced by the user.

Once the above parameters are loaded the custom software detects automatically the center of the focal spots by applying image processing techniques. For the identification and location 
of the focal spots, a threshold is applied to the sum of the reference image rows and columns, generating a reference grid pattern. Then, for each obtained reference spot the algorithm applies a threshold to the surrounding region and looks for objects (function regionprops [27]). If there is only one object inside the considered region, it is assigned to the corresponding reference spot. At the bottom of Fig. 4 the focal spots of the reference image (green points) and the focal spots of the aberrated image (red points) are shown. If the option "View/Points" is selected, the program marks the points that will be used in the calculation of the wavefront parameters. Moreover, if the option "View/Lines" is selected, the displacement of each focal spot will be shown as a line. By clicking the buttons + and - new focal spots, not detected with the automatic algorithm, can be added or deleted to form pairs of focal spots that are not properly matched up. Clicking the "Reset" button, the program erases all the focal spots selection and recalculates them automatically. The yellow circular line in Fig.4 shows the pupil considered in the calculations. It is determined by the best area for the calculation of the Zernike coefficients according to the selected focal spots.

\subsection{The calculation screen}

Figure 5 shows the central part of the GUI corresponding to the calculation screen. The "?” button opens a help window with information about the Zernike polynomials. By clicking the “CALCULATE" button, the corresponding Zernike coefficients are calculated from the displacement between the reference spots and the focal spots provided by the tested lens, i.e., the displacement vector $\boldsymbol{d}$ is obtained. Using Eq. (5), the Zernike coefficients are calculated up to $k=14$. Together with the $k$ parameter the parameters $n$ and $m$ are also shown, which represent the radial order and the azimuthal order of the corresponding Zernike mode [24]. As an output parameter, this window shows the numerical values of the computed Zernike coefficients (in microns). This screen shows the aberration corresponding to each Zernike polynomial mode (tilt, defocus, astigmatism, coma, ...). Additionally, the checkboxes of the modes allow to select the modes used in the wavefront reconstruction. Finally, the wavefront is reconstructed from the 
calculated Zernike coefficients using Eq. (2). By defaults, the modes $k=1$ and $k=2$ are unchecked in order to avoid the tilt effect of the system.

\subsection{The output screen}

The output screen (Fig. 6) shows the wavefront reconstruction corresponding to the values in Fig. 5. If the checkbox "Phase" is selected, the reconstructed wavefront is shown applying the modulus $\lambda$ function in order to stand out the phase shifts. Clicking the "Save" button, the GUI exports the following information about the reconstruction: An image file with the superposition of the reference and the aberrated focal spots, an image file with the wavefront reconstruction, and a data file with the calculated Zernike coefficients and the pupil radius. Additionally, the GUI shows the corresponding values under the conventional script notation in terms of sphere $(S)$, cylinder $(C)$, and angle $(\alpha)$, computed using Eq. (7) (see the bottom of Fig. 6). Note that in the example of Fig. 6, the analyzed wavefront corresponds to a negative lens with power -2.24 $\mathrm{D}$ and a nearly negligible astigmatism.

\section{Results}

Figure 7 shows the basic arrangement of the SH sensor developed in our laboratories. A point light source is generated with a red LED of wavelength $633 \mathrm{~nm}$ and a microscope objective. This point source is located on the focal plane of the lens $L 1$ to obtain a collimated planar wavefront. The tested lens is mounted on a rotary stage with an $\mathrm{XY}$ micrometric platform. The lenses $L 2$ and $L 3$ form a confocal system with the tested lens and the MLA at conjugated planes. In this customized SH sensor we used a $13 \times 13$ convergent MLA. Each microlens has a focal length of $f_{M L A}=50 \mathrm{~mm}$ and a pitch of $500 \mu \mathrm{m}$. An 8-bit CMOS camera with $1280 \times 980$ pixels (the effective size of the pixel is $4.4 \mu \mathrm{m}$ ) is located on a plane conjugated with the focal plane of the MLA. Finally, the captured image files are analyzed with the GUI described in the previous section.

To test the experimental setup and the program, a set of monofocal lenses (two concave, two convex, two cylindrical and two sphero-cylindrical lenses) were measured and compared 
with its nominal value (see Table 1). The axis has not been considered in the astigmatic lenses due to its dependence on the lens placing in each measure. Each lens was measured four times to determine the mean value of the sphere, the cylinder and their uncertainty. In all cases, the standard deviation of the measurements was lower than $0.01 \mathrm{D}$. Note that a good agreement is obtained between the experimental and nominal values (taking into account that the nominal values have an uncertainty of $0.12 \mathrm{D}$ according to the manufacturers).

Figure 8 shows the wavefront modulus $\lambda$ obtained from the calculated Zernike coefficients (without tilts and up to fourth radial order Zernike polynomials). The representation of the wavefront modulus $\lambda$ gives visual information about if the tested lens is spherical, cylindrical or sphero-cylindrical, or if the lens has low or high power and even which is the direction of the cylinder axis. In this way, we can determine from Fig. 7 that L1 and L2 are convergent lenses, and L2 has a higher power than L1. Note also that L3 and L4 are divergent lenses, and L4 has a higher power than L3. On the other hand, L5 and L6 are astigmatic lenses with different cylindrical power and orientation. Finally L7 and L8 are lenses with different combinations of spherical and cylindrical powers.

\section{Conclusions}

The wavefront sensors are an integral part of the subject Opto-Electronic Sensors Technology of the Master's Degree in Sensors for Industrial Applications at the Technical University of Valencia, Spain. The theoretical background of this topic is supplemented with theoretical lessons in the classroom and with laboratory work. Compared with more expensive commercial alternatives, the proposed open experimental set-up allows students to investigate the influence of each component of the system. To avoid a tedious computational work, the sensor response is assisted with the MATLAB GUI presented in this work. As a practical laboratory experience with students, we tested different monofocal lenses obtaining a very good agreement with the nominal values. Students thought it was very interesting and motivating, and the mathematical difficulties were overcame with the developed GUI. On the other hand, the 
experiment helped students to understand the basic principles of wavefront sensors and their applications in some scientific and technological areas. For example, the proposed setup can be used also to study the lasers beam quality.

\section{ACKNOWLEDGMENT}

This work was supported in part by the Ministerio de Economía y Competividad under grant FIS2011-23175, by Generalitat Valenciana under grant PROMETEOII/2014/072, and by the Universitat de València under grant UV-SFPIE_DINV14-222801. This work has been developed by the Teaching Innovation Groups from the Universitat Politècnica de València (MoMa) and the Universitat de València (GCI35/2009).

\section{REFERENCES}

[1] J. Marino and F. Wöger, Feasibility study of a layer-oriented wavefront sensor for solar Telescopes, Appl. Opt., Vol. 53, 2014, pp. 685-693.

[2] F. Feng, I. H. White, and T. D. Wilkinson, Aberration correction for free space optical communications using rectangular Zernike modal wavefront sensing, J. Lightwave Technol., Vol. 32, 2014, pp. 1239-1245.

[3] V. Micó, Z. Zalevsky, and J. Garcia, Superresolved common-path phase-shifting digital inline holographic microscopy using a spatial light modulator, Opt. Lett., Vol. 37, 2012, pp. 4988-4990.

[4] M. Paurisse, M. Hanna, F. Druon, and P. Georges, Wavefront control of a multicore ytterbium-doped pulse fiber amplifier by digital holography, Opt. Lett., Vol. 35, 2010, pp. 14281430.

[5] C. Huang, H. Huang, H. Toyoda, T. Inoue, and H. Liu, Correlation matching method for high-precision position detection of optical vortex using Shack-Hartmann wavefront sensor, Opt. Express, Vol. 20, 2012, pp. 26099-26109.

[6] F. Vega, F. Alba-Bueno, and M. S. Millan, Energy efficiency of a new trifocal intraocular lens, J. Eur. Opt. Soc.: Rapid Publications, Vol. 9, 2014, pp. 14002. 
[7] J. Fuentes-Fernández, S. Cuevas, L. C. Álvarez Nuñez, and A. Watson, Tests and evaluation of a variable focus liquid lens for curvature wavefront sensors in astronomy, App. opt., Vol. 52, 2013, pp. 7256-7264.

[8] V. Akondi, S. Castillo, and B. Vohnsen, Digital pyramid wavefront sensor with tunable modulation, Opt. Exp., Vol. 21, 2013, pp. 18261-18272.

[9] N. Gu, L. Huang, Z. Yang, and C. Rao. A single-shot common-path phasestepping radial shearing interferometer for wavefront measurements, Opt. Express, Vol. 19, 2011, pp. 47034713.

[10] K. Rahbar, K. Faez, and E. Attaran-Kakhki, Estimation of phase wavefront aberration distribution function using wavelet transform profilometry, App. Opt., Vol. 51, 2012, pp. 33803386.

[11] T. Ling, D. Liu, Y. Yang, L. Sun, C. Tian, and Y. Shen, Off-axis cyclic radial shearing interferometer for measurement of centrally blocked transient wavefront, Opt. Lett., Vol. 38, 2013, pp. 2493-2495.

[12] M. Idir, K. Kaznatcheev, G. Dovillaire, J. Legrand, and R. Rungsawang, A 2D high accuracy slope measuring system based on a Stitching Shack Hartmann Optical Head, Opt. Express, Vol. 22, 2014, pp. 2770-2781.

[13] A. Polo, V. Kutchoukov, F. Bociort, S. F. Pereira, and H. P. Urbach, Determination of wavefront structure for a Hartmann Wavefront Sensor using a phase-retrieval method, Opt. Express, Vol. 20, 2012, pp. 7822-7832

[14] B. C. Platt and R. Shack, History and principles of Shack-Hartmann wavefront sensing, $J$. Refract. Surg., Vol. 17, 2001, pp. S573-S577.

[15] C. Li, B. Li, and S. Zhang, Phase retrieval using a modified Shack-Hartmann wavefront sensor with defocus, App. Opt., Vol. 53, 2014, pp. 618-624.

[16] P. Migda, P. Fita, C. Radzewicz, and U. Mazurek, Wavefront sensor with Fresnel zone plates for use in an undergraduate laboratory, Am. J. Phys., Vol. 76, 2008, pp. 229-635.

[17] J. M. Ramirez-Cortes, V. Alarcon-Aquino, P. Gomez-Gil, A. Diaz-Mendez, M. IbarraBonilla, and I. García-Enriquez, Interactive educational tool for compensators design in 
MATLAB ${ }^{\circledR}$ using frequency response analysis, Comput. Appl. Eng. Educ., Vol. 22, 2014, pp. 699-707.

[18] R. Conan and C. Correira, Object-Oriented Matlab Adaptive Optics Toolbox, Proc. SPIE 9148, 91486C (1-17) (2014).

[19] Adaptive optics toolbox (2015) online at http://in.mathworks.com/products/connections/product_detail/product_35400.html Accessed 23 July 2015.

[20] Some instrumental work on the Shack-Hartmann wavefront sensor and its implementation for Vision Science (2015) online at http://optics.nuigalway.ie/people/charlie/main.html Accessed 23 July 2015.

[21] Wavefront optics toolbox (2015) online at http://white.stanford.edu/teach/index.php/Wavefront_optics_toolbox Accessed 23 July 2015. [22] Modal Shack-Hartmann wavefront sensor toolbox (2015) online at http://www.antonello.org/code.php Accessed 23 July 2015.

[23] V. N. Mahajan, Zernike circle polynomials and optical aberrations of systems with circular pupils, Appl. Opt. Vol. 33, 1994, pp. 8121-8124.

[24] L. N. Thibos, R. A. Applegate, J. T. Schwiegerling, and R. Webb, Standards for reporting the optical aberrations of eyes, J. Refract. Surg., Vol. 18, 2002, pp. 652-660.

[25] L. N. Thibos, X. Hong, A. Bradley, and R. A. Applegate, Accuracy and precision of objective refraction from wavefront aberrations, J. Vision, Vol. 4, 2004, pp. 329-351.

[26] L. N. Thibos, W. Wheeler, and D. Horner, Power Vectors: An application of Fourier analysis to the description and statistical analysis of refractive error, Optometry Vis. Sci., Vol. 74, 1997, pp. 367-375.

[27] Measure properties of image regions (2015) online at http://es.mathworks.com/help/images/ref/regionprops.html Accessed 23 July, 2015 


\begin{tabular}{|c|c|c|c|c|}
\hline \multirow{2}{*}{ Lens } & \multicolumn{2}{|c|}{ Nominal values (D) } & \multicolumn{2}{c|}{ Measured values (D) } \\
\cline { 2 - 5 } & Sphere & Cylinder & Sphere & Cylinder \\
\hline L1 & 0.25 & 0.00 & 0.23 & -0.02 \\
\hline L2 & 2.50 & 0.00 & 2.19 & -0.06 \\
\hline L3 & -0.25 & 0.00 & -0.24 & -0.01 \\
\hline L4 & -2.50 & 0.00 & -2.25 & -0.03 \\
\hline L5 & 0.00 & -0.50 & 0.02 & -0.44 \\
\hline L6 & 0.00 & -2.25 & 0.00 & -2.06 \\
\hline L7 & 1.25 & -2.00 & 1.07 & -1.75 \\
\hline L8 & -0.25 & -2 & -0.26 & -1.82 \\
\hline
\end{tabular}

Table 1: Comparison of the spherical and cylindrical powers measured for the tested lenses with their nominal values. 
a) Tested Wavefront

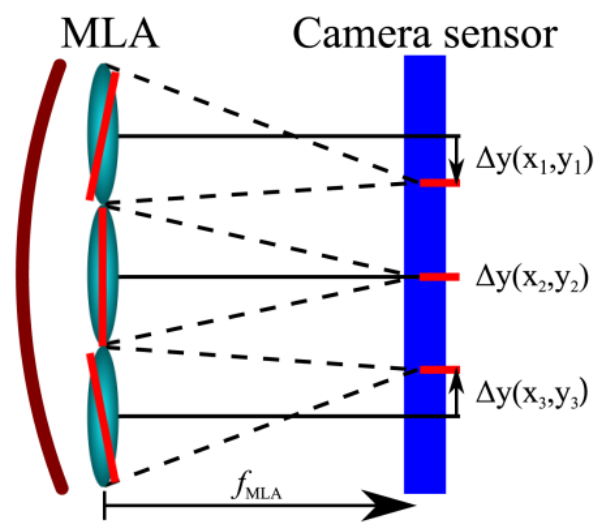

b) Sensor image

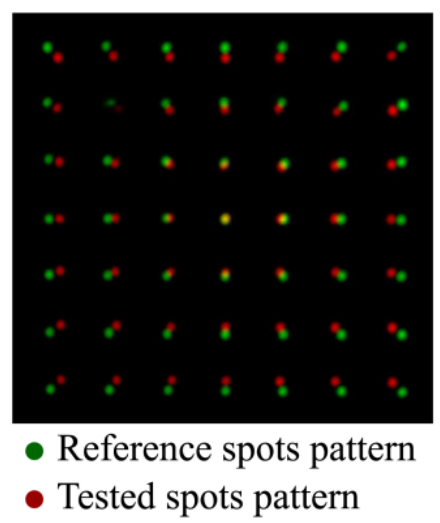

Figure 1: a) Basic elements of a SH. b) Superimposed images of a reference spots pattern and a tested spots pattern. If a plane wave or reference wavefront reach the MLA, each microlens give a reference spot (green spots). If the wavefront is aberrated, the tested spots are displaced (red spots). 


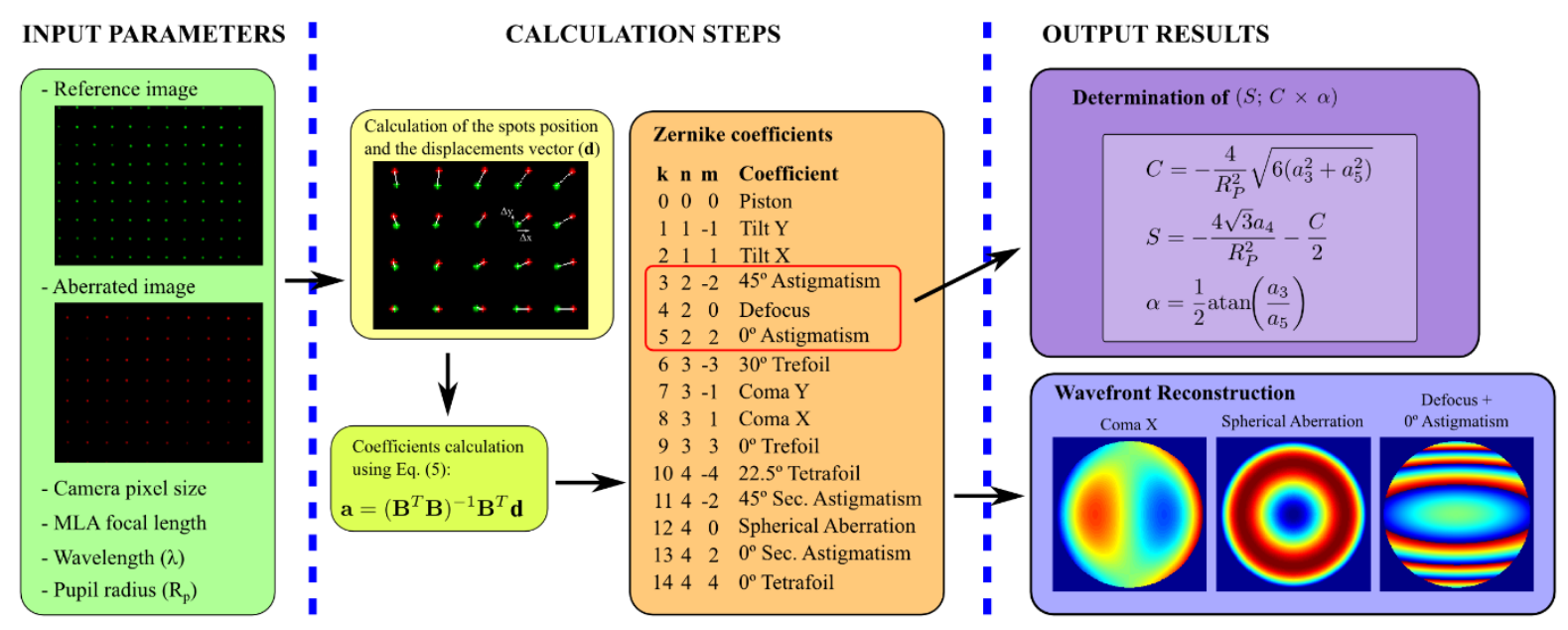

Figure 2: Flowchart of the proposed algorithm. 


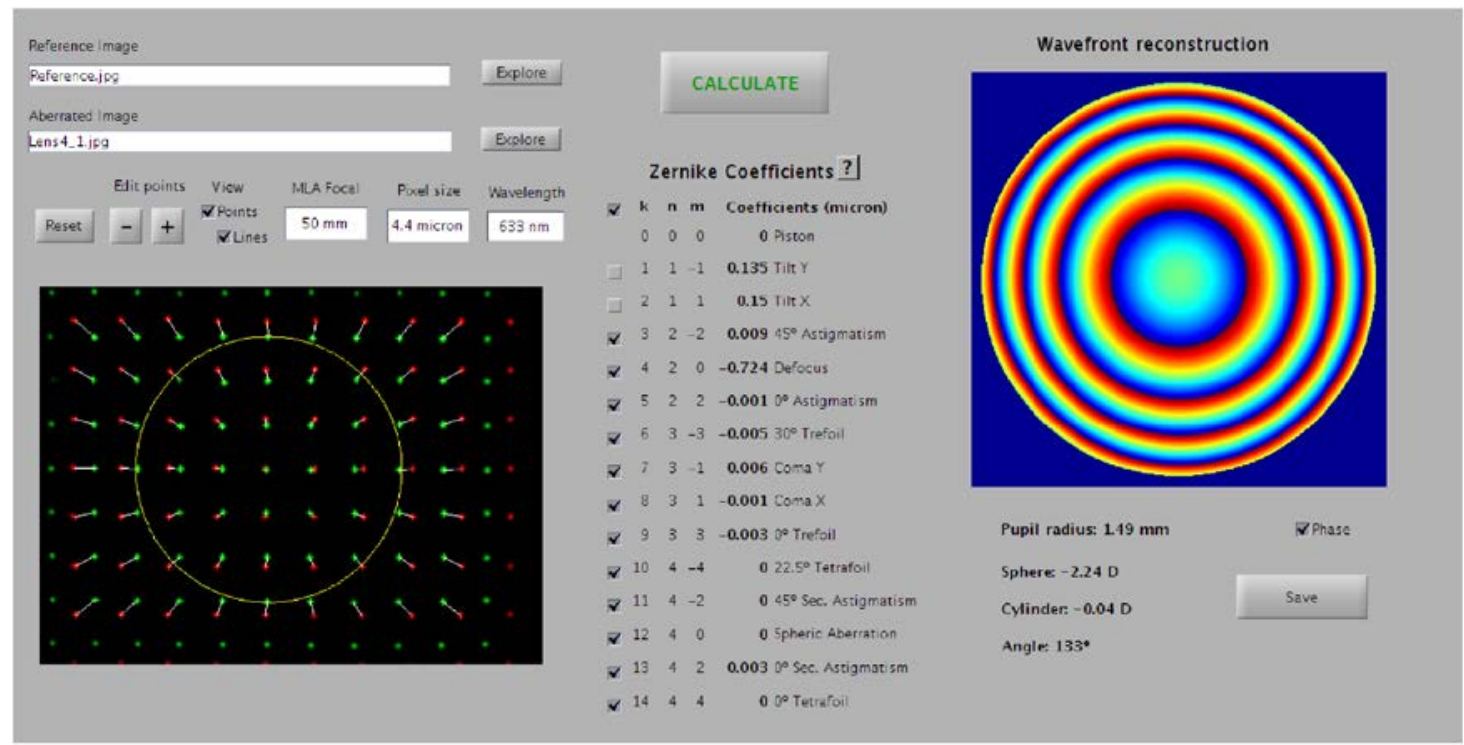

Figure 3: Main screen of the MATLAB GUI. 


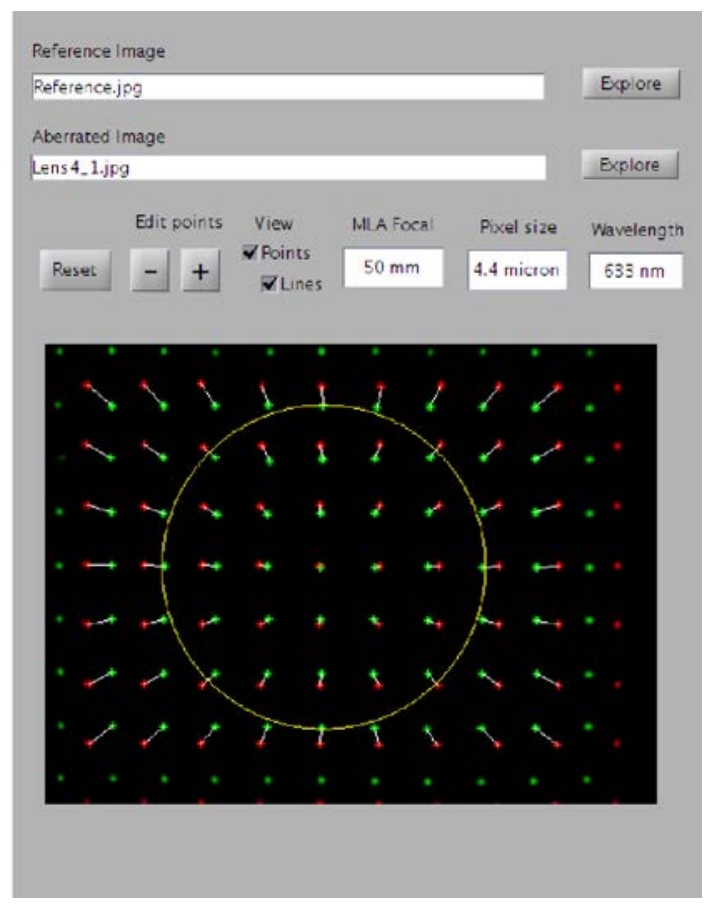

Figure 4: Input screen of the MATLAB GUI. 


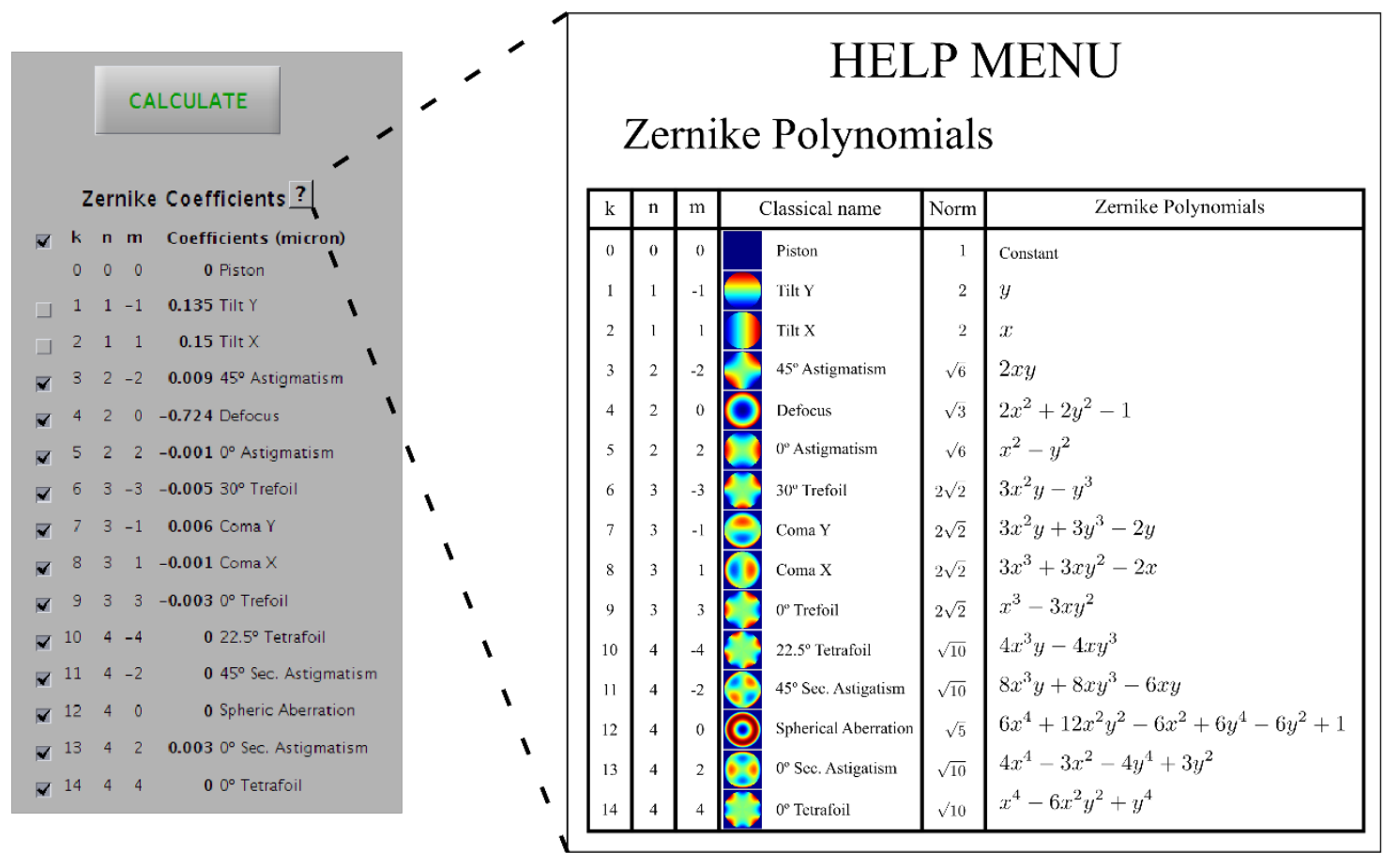

Figure 5. (Left) Calculation screen of the MATLAB GUI- (Right) and Help Menu about the

Zernike polynomials. 


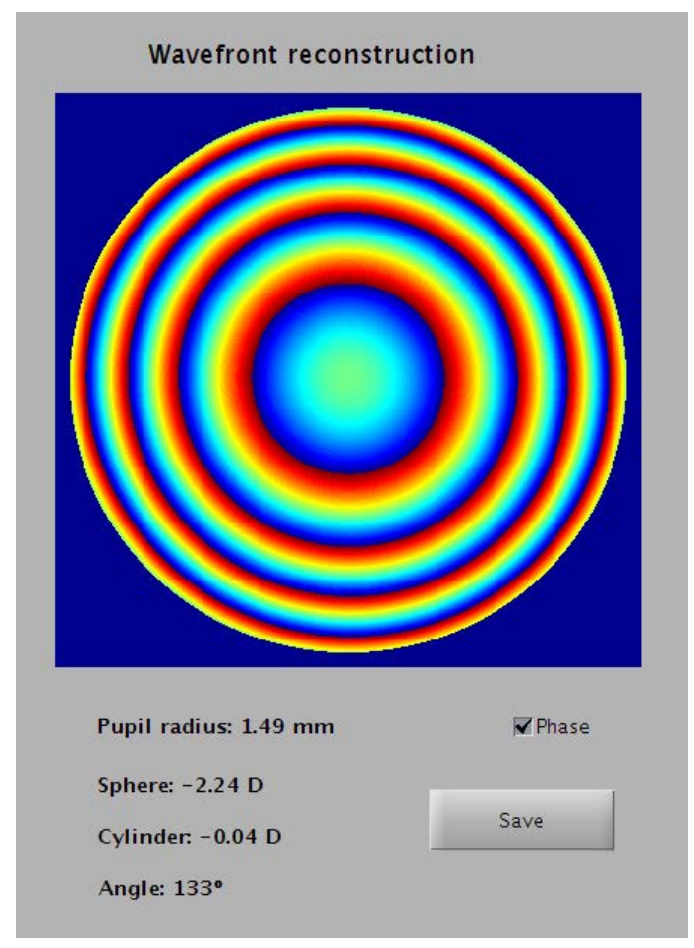

Figure 6. Output screen of the MATLAB GUI. 


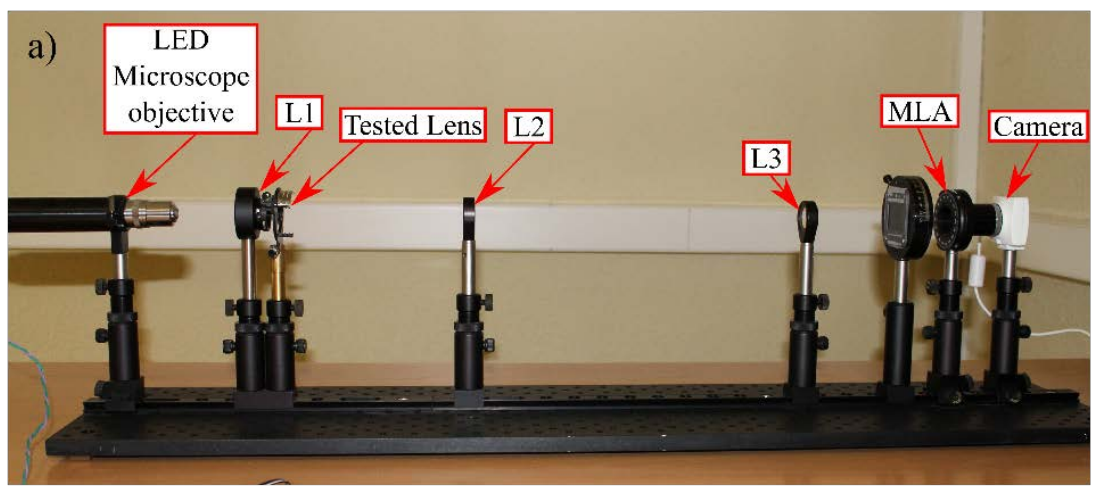

b)
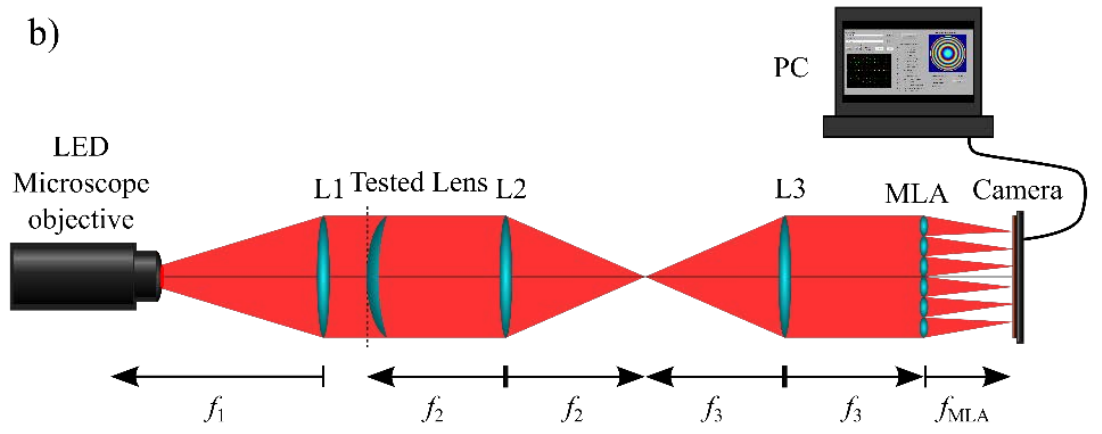

Figure 7: a) Experimental setup employed to study the aberrations of the tested lenses.

b) Experimental setup scheme. 

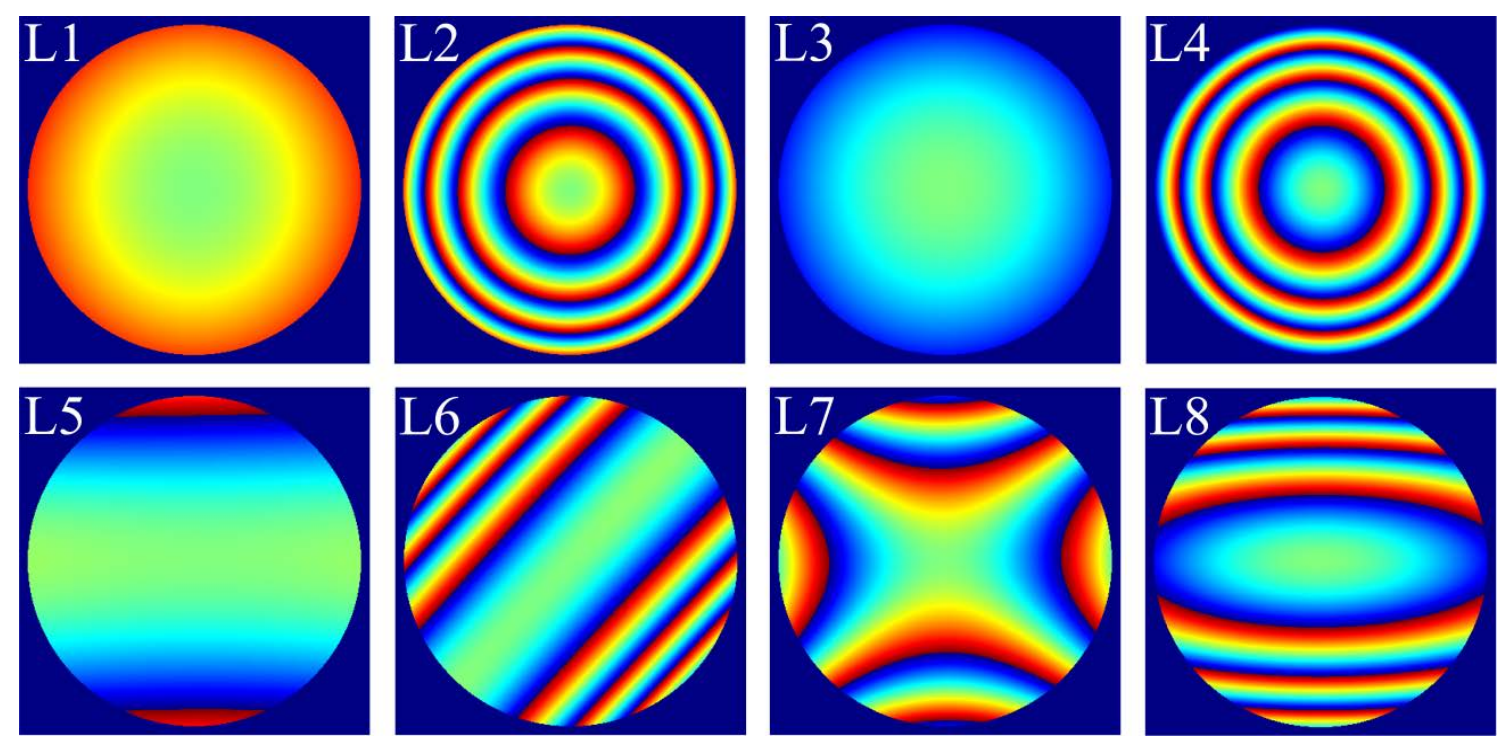

Figure 8: Wavefront modulus $\lambda$ for the different tested lenses. 